\title{
Demonstrating Noninferiority of Accelerated Radiotherapy With Panitumumab vs Standard Radiotherapy With Cisplatin in Locoregionally Advanced Squamous Cell Head and Neck Carcinoma
}

\author{
David Cheng, AM, \\ Department of Biostatistics, Harvard T. H. Chan School of Public Health, Boston, Massachusetts \\ Kyongsun Pak, BPharm, \\ Department of Clinical Medicine (Biostatistics), Kitasato University School of Pharmacy, Minato- \\ ku, Tokyo, Japan \\ Lee-Jen Wei, PhD \\ Department of Biostatistics, Harvard T. H. Chan School of Public Health, Boston, Massachusetts
}

To the Editor Siu and colleagues ${ }^{1}$ conducted the largest clinical trial to date to assess accelerated-fractionation radiotherapy (AFX) plus panitumumab compared with standardfractionation radiotherapy (SFX) plus cisplatin. The study was designed for a superiority analysis to detect a difference in the progression-free survival (PFS) that corresponds to a hazard ratio (HR) of 0.70 , requiring a total of 246 events and 320 patients to achieve appropriate statistical power. If superiority was not demonstrated, the study tested noninferiority of AFX plus panitumumab to SFX plus cisplatin with a noninferiority margin of 1.15. The study was unable to demonstrate either the superiority or noninferiority of AFX plus panitumumab to SFX plus cisplatin.

There are several issues regarding the design and analysis. It is unclear how to justify the statistical validity of the aforementioned 2-stage testing procedure (superiority first and then noninferiority) such that the overall false-positive rate is less than 5\%. Moreover, with a noninferiority margin of $1.15,1650$ events would be needed by the end of the study to assess noninferiority with appropriate power. ${ }^{2}$ Achieving noninferiority would be nearly impossible in a study with 320 patients. The results reported that a total of 93 PFS events occurred among the 320 patients, with 43 events occurring among those receiving AFX plus panitumumab and 50 among those receiving SFX plus cisplatin. The confidence interval (CI) for the HR (AFX plus panitumumab vs SFX plus cisplatin) was 0.60 to 1.50 , which is the basis for the inability to demonstrate either superiority or noninferiority. The large CI would be interpreted as indicating that the study lacks adequate power for either setting.

Corresponding Author: Lee-Jen Wei, PhD, Department of Biostatistics, Harvard T. H. Chan School of Public Health, 655 Huntington Ave, Boston, MA 02115 (wei@ hsph.harvard.edu).

Conflict of Interest Disclosures: None reported. 
The precision of the HR estimate depends only on the observed number of events, not on patients' exposure times. This is the main reason that 1650 events would be needed to reach the prespecified noninferiority margin. Quantifying treatment differences based on the HR is also difficult to interpret clinically. ${ }^{3,4}$ An alternative approach to evaluate noninferiority is to use the restricted mean PFS time. ${ }^{3,4}$ Based on data scanned ${ }^{4,5}$ from Figure 2, the upper bound for the CI for the difference of the 36-month restricted mean PFS time for SFX plus cisplatin vs AFX plus panitumumab was 1.7 months. This suggests that, in the worst case, patients receiving AFX plus panitumumab have 1.7 months shorter PFS time on average than patients receiving SFX plus cisplatin, which is less than 5\% of 36 months. This quantification provides a heuristically, clinically interpretable treatment effect for noninferiority assessment. Using this measure, it would not require an unfeasible study size to achieve adequate power. We recommend considering this alternative for future studies with event time observations.

\section{References}

1. Siu LL, Waldron JN, Chen BE, et al. Effect of standard radiotherapy with cisplatin vs accelerated radiotherapy with panitumumab in locoregionally advanced squamous cell head and neck carcinoma: a randomized clinical trial. JAMA Oncol. 2017;3(2):220-226. [PubMed: 27930762]

2. Leyland-Jones B, Bondarenko I, Nemsadze G, et al. A randomized, open-label, multicenter, phase III study of epoetin alfa vs best standard of care in anemic patients with metastatic breast cancer receiving standard chemotherapy. J Clin Oncol. 2016;34(11):1197-1207 [PubMed: 26858335]

3. Uno H, Wittes J, Fu H, et al. Alternatives to hazard ratios for comparing the efficacy or safety of therapies in noninferiority studies. Ann Intern Med. 2015; 163(2):127-134. [PubMed: 26054047]

4. Uno H, Claggett B, Tian L, et al. Moving beyond the hazard ratio in quantifying the between-group difference in survival analysis. J Clin Oncol. 2014;32(22):2380-2385. [PubMed: 24982461]

5. Guyot P, Ades AE, Ouwens MJ, Welton NJ. Enhanced secondary analysis of survival data: reconstructing the data from published Kaplan-Meier survival curves. BMC Med Res Methodol. 2012;12:9. [PubMed: 22297116] 